\title{
On the Development of a Personalized Augmented Reality Spatial Ability Training Mobile Application
}

\author{
Christos PAPAKOSTAS ${ }^{1}$, Christos TROUSSAS, Akrivi KROUSKA, Cleo \\ SGOUROPOULOU \\ Department of Informatics and Computer Engineering \\ University of West Attica, Greece
}

\begin{abstract}
Augmented Reality has been integrated in educational settings in the field of engineering. Prior research has examined the learning outcomes and the pedagogical affordances of this technology. However, training undergraduate engineers, from diverse knowledge level, requires customized training approach, tailored to the individual learning pace. In this paper, we present PARSAT (Personalized Augmented Reality Spatial Ability Training), which is a mobile Augmented Reality application for the enhancement of students' spatial visualization skills. The application takes into account the theoretical contents of engineering design, deployed through video tutorials, and student-computer interaction with 3D objects. Students interpret different views of a 3D object, which are represented on their mobile screen. PARSAT efficaciously strengthens students' recognition of spatial structures and views, adjusted to the fulfillment of their personal needs. In terms of personalization, PARSAT consists of different levels, which do not follow a linear flow, as each student takes part in a different sequence of activities, according to their time spent in the 3D object manipulation, and their assessment scores at the end of each level. Furthermore, an agent is used to analyze students' knowledge level, and send them feedback. The system reduces unnecessary cognitive load and, at the same time, improves students learning experience in learning engineering drawing.
\end{abstract}

Keywords. Spatial ability, personalized augmented reality, mobile application, customized training, machine learning

\section{Introduction}

Spatial ability, as a factor of human intelligence, was initially recognized and studied by Thorndike [1]. Thorndike's proposed model consisted of three components, namely abstract, mechanical and social intelligence, and served as the early stage research for later studies on spatial ability. Thurstone [2] suggested that the intelligence consisted of seven primary mental abilities, one of which is spatial visualization, as involved in visualizing and manipulating objects. Thurstone [3] defined three core components of

\footnotetext{
${ }^{1}$ Corresponding Author; E-mail: cpapakostas@uniwa.gr.
} 
spatial ability, namely mental rotation, spatial visualization, and spatial perception. Mental rotation is defined as the ability to recognize an object being moved in different directions or angles; spatial visualization is defined as the ability to recognize the parts of an object when it is moved or displaced from its original position; and spatial perception is defined as the ability to use the own body orientation to interact with the environment and, therefore, with spatial orientation.

Gardner [4] introduced the concept of multiple intelligences, rather than defining intelligence as a single general ability. Gardner's argument stated that there is a wide range of cognitive abilities, not necessarily correlated between them, namely musicrhythmic and harmonic, visual-spatial, linguistic-verbal, logical-mathematical, bodilykinesthetic, interpersonal, intrapersonal, and naturalistic. Many modern theories proposed additional factors or cognitive processes, in their effort to better understand spatial ability $[5,6]$. Each research has added significantly to the definition of spatial ability, as a form of intelligence, where a person demonstrates the capacity to mentally generate, transform, and rotate a visual image and thus, understand and recall spatial relationships between real and imagined objects.

Smith [7] reported a list of 84 different occupations requiring top-level spatial ability, 26 of which were related to engineering, while 14 of them were related to graphics. There is significant correlation between spatial ability and many scientific fields, such as geometry, physics and technical drawing. Field [8] designed a 52-hours course aiming to develop skills in representing spatially visualized objects through projections. Martín - Dorta et al. [9] also launched a fast remedial course based on 3-dimensional modelling for improving spatial abilities of engineering students. Designing 3dimensional objects is highly considered to be a crucial factor in the development of spatial skills $[10,11]$.

However, many students find difficulties in visualizing abstract concepts or the geometry of 3-dimensional objects [12]. The training of spatial skills is either based on the manipulation of physical models used for exercises, or modelling in computer software [13]. Zaretsky \& Bar [14] positively introduced virtual reality (VR) for object rotation training, and respectively with the increased duration that they observed in students' concentration, they finally enhanced their academic achievements. Overall, the presentation of an object in 3-dimensional view using a smartphone or a tablet, can help students having difficulties to understand its geometry, to watch it from different angles. Hidden details of the object are revealed due to the software's availability for various views orientations, and the technical drawing can be implemented faster.

Apart from VR, Augmented Reality (AR) is another alternative technology for spatial skills training, considered by many recent studies as the best alternative teaching approach [15-18]. AR superimposes a computer-generated image on a user's view of the real world, thus providing a composite view. Azuma et al. [19] defined AR as a system which a) combines real and virtual objects in a real environment; b) runs interactively in real time; and c) aligns real and virtual objects with each other. The evaluation of AR in educational settings is gaining more place, mainly due to the emergence of smartphones and tablets. AR has a great potential in enhancing students' spatial ability and learning experience.

A few researchers have focused on the integration of mobile AR in spatial ability training. Tumkor [20] tested mixed reality (MR) technologies for more than 3 years in engineering design courses, representing two-dimensional sketches in front of students, while also rotating the virtual objects as they wished. The results reported an overall improvement of students' visualization skills. Figueiredo et al. [21] presented EducHolo, 
a mobile AR learning tool for the visualization and interaction of 3D models, providing students with a better perception of models and improving theirs sketching at 2D orthographic views. De Ravé et al. [22] designed DiedricAR, a mobile AR exercise workbook, aimed at the learning of descriptive geometry and explored the application's benefits for students' spatial ability. Kaur et al. [23] designed and developed GeoSolvAR, an AR-based solution for visualizing 3D solids. Omar et al. [24] emphasized on the development of visualization skills and concept understanding, by teaching and learning orthographic projections using an AR engineering drawing application, namely AREDApps. Papakostas et al. [25] explored the advantages and trends of AR in spatial ability training based on the revision of articles over the decade 2010-2019. The trend, in the development of AR applications, is to use three-dimensional objects towards improving spatial ability. The considerable findings identified the lack of personalized applications adapting their content to the learner's level of visualization skills. That is an area where there is still vast potential development.

To address this problem, we designed a mobile personalized AR spatial ability training application, named PARSAT, which is tailored to meet the user's spatial skills level. The diversity of the characteristics that each student presents, such as the level of knowledge, the gender, the learning style, etc, define the process of learning. The novelty of our research lies on the introduction of the concept of adaptive AR, as the nextgeneration advanced educational application, designed to improve students' visualization skills. Adaptive AR is among the most promising technologies in teaching and learning processes, enabling the students to benefit from the relations with the 3D objects in the space, and thus, keeping pace with their own abilities and interest.

\section{Overview of the system}

The main difference, between PARSAT and the existing AR applications, is that the educational process is adaptive [26-28]. The basic idea of the application is to deliver relevant content based, at first, on student's background knowledge and secondly, on student's assessment score [29-31].

The application consists of three different stages, each one covering terms of the Orthographic Projection chapter of engineering design graphics course. The most fundamental technique is called graphical projection, by which a three dimensional object is represented on a planar surface. Orthographic projection uses a series of twodimensional views, arranged in a standard manner, in order to fully document the object's geometry [32].

Students can receive a short, medium or long tutorial at the beginning of the training, depending on their skill level and past knowledge. PARSAT then assigns each student the alternative learning path that maximizes his/her target key performance indicator (KPI). 
In order to achieve the research goal, a framework has been developed reflecting the typical spatial abilities training, comprising of three modules which are: i) the User Interface Model (UIM); ii) the Domain Knowledge Model (DKM); and iii) the Personalization Model (PM), analyzed at the next subsections. Figure 1 illustrates the logical architecture of PARSAT.

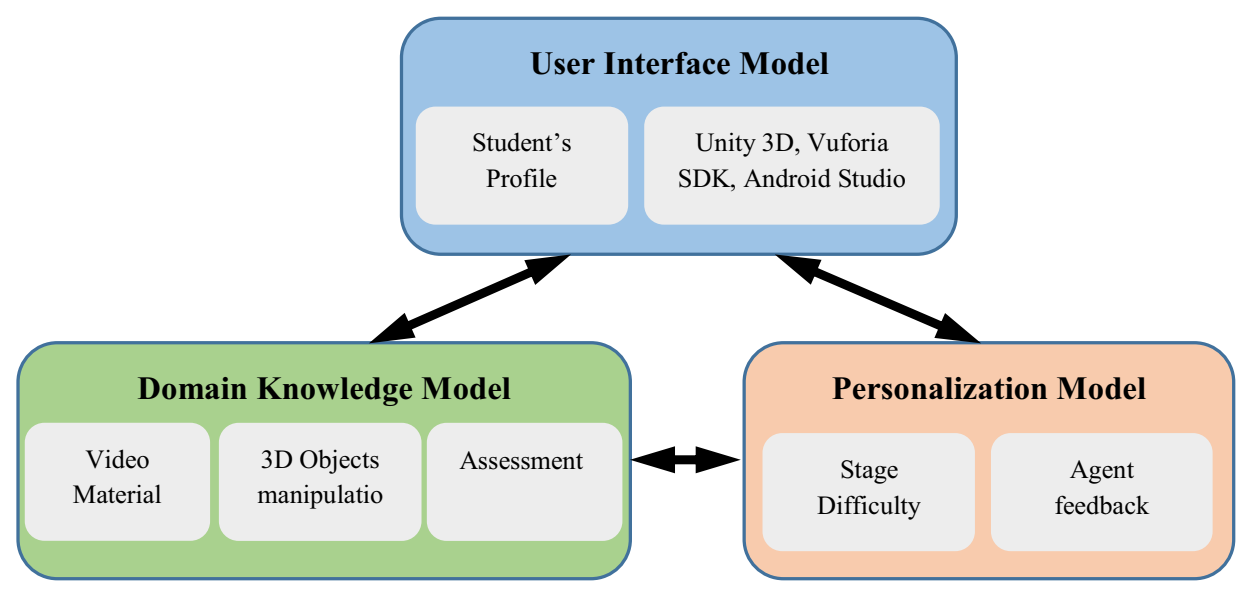

Figure 1. Logical architecture of PARSAT

\subsection{User interface model}

The user interface model includes the context and the function of the AR application, making the student experience as clean and simple as possible. As such, students create their profile, based on the demographic characteristics of age and gender, and cognitive characteristics of prior knowledge of the topic, before training starts.

One of the key difficulties in developing AR applications is the tracking of the user's viewpoint. The application has to know all of the time where the user is looking in the real world, using the mobile's rear-view camera. To this direction, we used Vuforia $^{2}$ Software Development Kit (SDK), one of the best-known AR tool sets, adding advanced computer vision functionality to both Android and iOS applications, and creating AR experiences that realistically interact with the 3D geometrical objects displayed at each stage. We took advantage of Vuforia's marker-based recognition and imported 3D objects with virtual buttons embedded, so that the students can move, rotate and zoom them.

Regarding the development framework, we used Unity $3 \mathrm{D}^{3}$ cross-platform game engine. In 2016, Vuforia announced a partnership with Unity to integrate the Vuforia platform into Unity's popular game engine and development platform. One of Unity's most powerful features is the extensible editor it has. We used editor scripting in C-Sharp

\footnotetext{
2 https://developer.vuforia.com/

3 https://unity.com/
} 
(C\#) programming language to extend the functionalities of the application. Finally, Android Studio Integrated Development Environment (IDE) is interfaced with Unity 3D to build the application for Android environment.

\subsection{Domain knowledge model}

The domain knowledge model includes the basic, intermediate and advanced topics from the domain of engineering graphics in an undergraduate level of the School of Engineering. Each one of the three levels of complexity consists of 5 sections (Table 1).

Table 1. Domain knowledge of PARSAT

\begin{tabular}{lll}
\hline Difficulty Level & \multicolumn{1}{c}{ Sections } \\
\hline Basic & 1. & Orthographic projection introduction \\
2. & The six principal views \\
3. & The glass box method \\
4. & Standard views - view alignment \\
5. & Line type - line weight \\
6. & Creating an orthographic projection \\
7ntermediate & 8. & Drawing an orthographic projection \\
& 9. & Parts of dimensions \\
10. & Scales \\
Advanced & 11. & Cutting plane \\
12. & Cutting plane line \\
13. & Section lining \\
14. & Full sections \\
15. & Half sections \\
\hline
\end{tabular}

The aforementioned sections, covered by PARSAT, are deployed firstly through recorded video tutorials, and secondly through interaction with augmented 3D objects, which were made in 3D software. Among a variety of 3D designing tools, we selected Autodesk 3D Studio $\mathrm{Max}^{4}$, as it is a product more suitable for modeling, architecture designs, engineering, and construction. All 3D models, created in 3D Studio Max, were exported to Unity using an appropriate generic file format.

The video tutorials have different details and length providing the alternatives of short, medium, and long tutorial, according to the student's background knowledge level.

\subsection{Personalization model}

The adaptation, or else personalization, model is modeling the users and customizes the components of the learning content and the flow of the learning. Each student follows a different sequence of activities that allows them to acquire knowledge and reach the topic's objectives.

\footnotetext{
${ }^{4}$ https://www.autodesk.com/products/3ds-max
} 
Each student's default starting stage is the second one, and a Difficulty Personalization module (DPM) defines whether the difficulty should be easier or harder for the student. Therefore, each student is either less frustrated, in case of a hard level, or less bored, in case the current level is too easy. DPM takes a set of inputs, such as firstly the time spent by the student on manipulating augmented 3D objects, and secondly the scores in each level's assessment, and derives the order of the stages delivered to the students.

As far as the evaluation is concerned, we used an agent to analyze the assessment score and send feedback to the user. Agent could help the student identify potential gaps in the knowledge domain, and proposes specific sections that should be repeated and/or book pages that should be thoroughly studied. Moreover, software agents could also enhance students' support level, by encouraging them to keep up the good effort.

\section{Evaluation}

The first task in evaluating the prototype, is to ask the target users for initial feedback on design, usability, and user experience. Towards this direction, 35 postgraduate students were recruited to test the application on their own. The participants were all students of a Master Degree program on Computer Science and Engineering of the Department of Informatics and Computer Engineering. The participants were asked to operate the PARSAT by themselves, to watch the video tutorials, to rotate the $3 \mathrm{D}$ objects in order to visualize and understand their structures, and finally took the spatial skills test to evaluate the training effect.

After interacting with the AR application, the participants answered a questionnaire, in order to determine their personal feelings about the training. All the items of the questionnaire (Table 2) were measured using a 7-point Likert scale ranging from (1) strongly disagree to (7) strongly agree.

Table 2. Questions asked about PARSAT

\begin{tabular}{lll}
\hline Constructs & & \multicolumn{1}{c}{ Question } \\
\hline Design & 1. & PARSAT has a simple and understandable menu \\
& 2. & PARSAT has good functionality \\
Usability & 3. & PARSAT presents high quality videos and 3D objects \\
& 4. & Using PARSAT enhances my spatial skills \\
& 5. & Using PARSAT increases my educational performance \\
User experience & 6. & I find PARSAT useful for my course \\
& 7. & I would like to use PARSAT in more knowledge sections \\
& 8. & I will recommend others to use PARSAT \\
& 9. & AR has become one of my favorite technologies \\
\hline
\end{tabular}

Descriptive analysis of mean and standard deviation was used to analyze the feedback towards using PARSAT. Other than that, short interview sessions were used, discussing the participants' experience with the system. Usability and system performance were measured.

In the following paragraph we briefly report our findings regarding the framework application. Based on the results gathered, all participants agreed that PARSAT had a 
positive training effect (mean level of agreement $=6.1 ; \mathrm{SD}=0.62$; score from 1 to 7 ). The participants strongly agreed that the AR integration allowed them to manipulate the 3D objects, improving their understanding of their geometry, thus the adaptive behavior of PARSAT significantly reduced the cognitive load.

In the rest of this section, we present some qualitative results, collected through opinion surveys and interviews with the participants (Figure 2). Three group interviews were carried out, and they described the application as challenging, the 3D objects very attractive and appropriate, and they craved for more teaching material. In the individual interviews, participants showed satisfaction with the use of these 3D tools during the course.

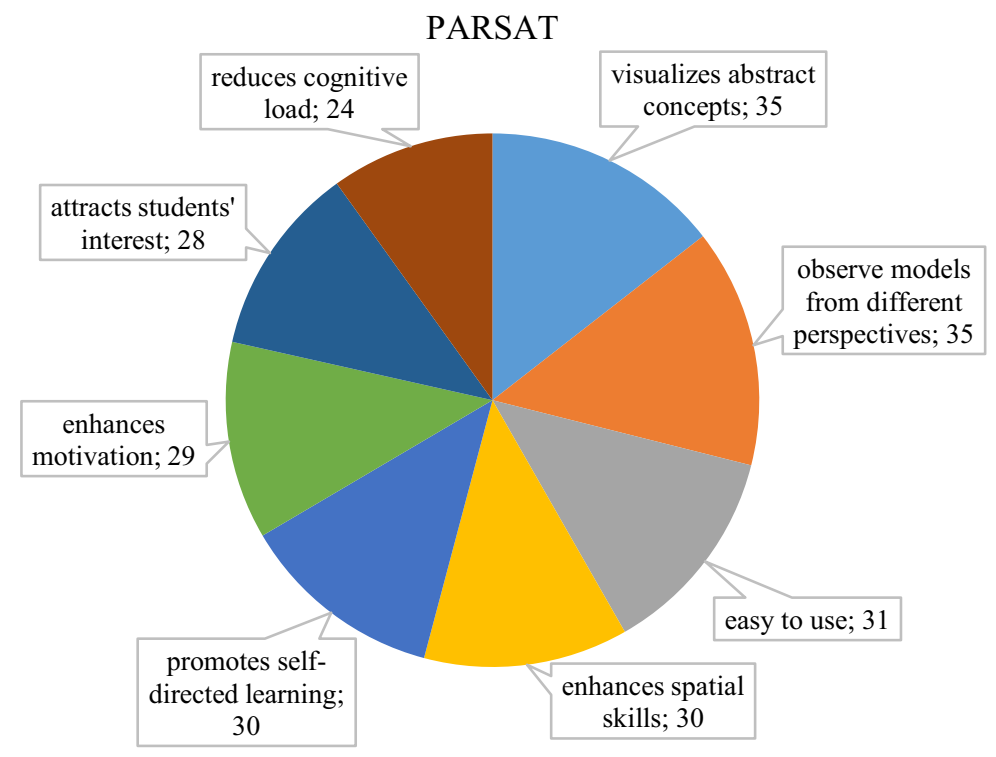

Figure 2. Learners' outcome of PARSAT

\section{Conclusion}

AR can currently help towards the digitalization of education, and the enhancement of learning achievements. To this direction, this paper focused on the development of PARSAT, which is a mobile AR application incorporating personalization for the enhancement of learners' visualization skills.

The findings of this research show that there is a significant difference in learners' spatial skills after using PARSAT application. PARSAT's optimizations are personalized and dynamic for each student, based on their state and progression. The adaptive process is critical for students' retention, and depends on the tuning of key parameters, such as the difficulty of the stages and the tutorial duration. Once there is enough collected data, PARSAT can select alternative for the student by a machine learning model. 


\section{Future work}

Our 3D object controller has only a rotate mode, while next version of PARSAT will embed zoom and move mode. Furthermore, the three stages of difficulty will be extended to five, so that each level will have less learning goals and students will not feel stressed about the cognitive load. Lastly, personalization, based on machine learning algorithm implemented in $\mathrm{C \#}$ script, is a long process that requires input data from a lot of participants, in order to train the model. So, we will incorporate the improvements in PARSAT and distribute it to engineering students' classrooms for training.

\section{References}

[1] E.L. Thorndike, On the Organization of Intellect., Psychol. Rev. 28 (1921), 141-151. https://doi.org/10.1037/h0070821.

[2] L.L. Thurstone, Primary mental abilities, Psychometric Monograph 1, Chicago, IL: Univer, 1938.

[3] L. Thurstone, Some Primary Abilities in Visual Thinking, Proc. Am. Philos. Soc. 94 (1950), 517-521.

[4] H. Gardner, Frames of mind: the theory of multiple intelligences, Basic Books, New York, 1983.

[5] J.W. Pellegrino, E.B. Hunt, Cognitive models for understanding and assessing spatial abilities, Intell. Reconceptualization Meas. (1991), 203-225.

[6] J.B. Carroll, Human cognitive abilities: A survey of factor-analytic studies, Cambridge University Press, 1993.

[7] I.M. Smith, Spatial ability : its educational and social significance, R.R. Knapp, San Diego, Calif., 1964.

[8] B.W. Field, A course in spatial visualization. Proceedings of the 6th International Conference on Engineering Design Graphics and Descriptive Geometry (1994), 257-261.

[9] N. Martín-Dorta, J.L. Saorín, M. Contero, Development of a fast remedial course to improve the spatial abilities of engineering students, J. Eng. Educ. 97 (2008), 505-513.

[10] A. Šafhalter, S. Glodež, A. Šorgo, M. Ploj Virtič, Development of spatial thinking abilities in engineering 3D modeling course aimed at lower secondary students, Int. J. Technol. Des. Educ. (2020). https://doi.org/10.1007/s10798-020-09597-8.

[11] R.H. McKim, Experiences in visual thinking, Brooks/Cole Pub. Co., Monterey, Calif., 1980.

[12] A.R. Arslan, S. Dazkir, Technical Drafting and Mental Visualization in Interior Architecture Education, Int. J. Scholarsh. Teach. Learn. 11 (2017). https://doi.org/10.20429/ijsotl.2017.110215.

[13] H.B.P. Gerson, S.A. Sorby, A. Wysocki, B.J. Baartmans, The development and assessment of multimedia software for improving 3-D spatial visualization skills, Comput. Appl. Eng. Educ. 9 (2001), 105-113.

[14] E. Zaretsky, V. Bar, Intelligent virtual reality and its impact on spatial skills and academic achievements, 10th Int. Conf. Inf. Syst. Anal. Synth. (2004), 107-113.

[15] O. Huerta, A. Kus, E. Unver, R. Arslan, M. Dawood, M. Kofoğlu, V. Ivanov, A design-based approach to enhancing technical drawing skills in design and engineering education using VR and AR tools, VISIGRAPP 2019 - Proc. 14th Int. Jt. Conf. Comput. Vision, Imaging Comput. Graph. Theory Appl. 3 (2019), 306-313. https://doi.org/10.5220/0007566003060313.

[16] R.M. Yilmaz, Augmented Reality Trends in Education between 2016 and 2017 Years, State Art Virtual Real. Augment. Real. Knowhow. (2018). https://doi.org/10.5772/intechopen.74943.

[17] C. Papakostas, C. Troussas, A. Krouska, C. Sgouropoulou, User acceptance of augmented reality welding simulator in engineering training, Educ. Inf. Technol. (2021). https://doi.org/10.1007/s10639-020-104187.

[18] C. Papakostas, C. Troussas, A. Krouska, C. Sgouropoulou, Measuring User Experience, Usability and Interactivity of a Personalized Mobile Augmented Reality Training System, Sensors. 21 (2021). https://doi.org/10.3390/s21113888.

[19] R. Azuma, Y. Baillot, R. Behringer, S. Feiner, S. Julier, B. MacIntyre, Recent advances in augmented reality, IEEE Comput. Graph. Appl. 21 (2001), 34-47. https://doi.org/10.1109/38.963459.

[20] S. Tumkor, Personalization of engineering education with the mixed reality mobile applications, Comput. Appl. Eng. Educ. 26 (2018), 1734-1741. https://doi.org/10.1002/cae.21942.

[21] M. Figueiredo, P.J.S. Cardoso, J.M.F. Rodrigues, R. Alves, Learning Technical Drawing with Augmented Reality and Holograms, Recent Adv. Educ. Technol. Methodol. (2014), 1-20.

[22] E.G. de Ravé, F.J. Jiménez-Hornero, A.B. Ariza-Villaverde, J. Taguas-Ruiz, DiedricAR: a mobile augmented reality system designed for the ubiquitous descriptive geometry learning, Multimed. Tools Appl. 75 (2016), 9641-9663. https://doi.org/10.1007/s11042-016-3384-4. 
[23] N. Kaur, R. Pathan, U. Khwaja, S. Murthy, GeoSolvAR: Augmented reality based solution for visualizing 3D Solids, Proc. - IEEE 18th Int. Conf. Adv. Learn. Technol. ICALT 2018 (2018), 372-376. https://doi.org/10.1109/ICALT.2018.00093.

[24] M. Omar, D.F. Ali, A.N. Nasir, M.S. Sunar, AREDApps: Integrating mobile augmented reality in orthographic projection teaching and learning, Int. J. Recent Technol. Eng. 8 (2019), 821-825.

[25] C. Papakostas, C. Troussas, A. Krouska, C. Sgouropoulou, Exploration of Augmented Reality in Spatial Abilities Training: A Systematic Literature Review for the Last Decade, Informatics Educ. 20 (2021), 107-130. https://doi.org/10.15388/infedu.2021.06.

[26] A. Krouska, C. Troussas, M. Virvou, K. Espinosa, C. Troussas, J. Caro, M. Virvou, Language Learning Assisted by Group Profiling in Social Networks, Int. J. Emerg. Technol. Learn. 8 (2013), 35-38. http://dx.doi.org/10.3991/ijet.v8i3.2684.

[27] C. Troussas, A. Krouska, and M. Virvou, Using a Multi Module Model for Learning Analytics to Predict Learners' Cognitive States and Provide Tailored Learning Pathways and Assessment, Virvou M., Alepis E., Tsihrintzis G., Jain L. (eds) Machine Learning Paradigms, Intelligent Systems Reference Library 158 (2020), Springer, Cham. https://doi.org/10.1007/978-3-030-13743-4 2.

[28] A. Krouska, C. Troussas, M. Virvou, Computerized Adaptive Assessment Using Accumulative Learning Activities Based on Revised Bloom's Taxonomy, Virvou M., Kumeno F., Oikonomou K. (eds) Knowledge-Based Software Engineering: 2018. JCKBSE 2018. Smart Innovation, Systems and Technologies 108 (2019), Springer, Cham. https://doi.org/10.1007/978-3-319-97679-2_26

[29] C. Troussas, A. Krouska, C. Sgouropoulou, Collaboration and fuzzy-modeled personalization for mobile game-based learning in higher education, Comput. Educ. 144 (2019), 103698. https://doi.org/10.1016/j.compedu.2020.103698.

[30] A. Krouska, C. Troussas, M. Virvou, Social networks as a learning environment: Developed applications and comparative analysis, 8th International Conference on Information, Intelligence, Systems \& Applications (IISA) (2017), 1-6. https://doi.org/10.1109/IISA.2017.8316430.

[31] C. Troussas, A. Krouska, C. Sgouropoulou, I. Voyiatzis, Ensemble Learning Using Fuzzy Weights to Improve Learning Style Identification for Adapted Instructional Routines, Entropy 22 (2020), 735. https://doi.org/10.3390/e22070735.

[32] J.D. Camba, J. Otey, M. Contero, M. Alcaniz, Visualization and Engineering Design Graphics with Augmented Reality, SDC Publications, Seiten, 2013. 